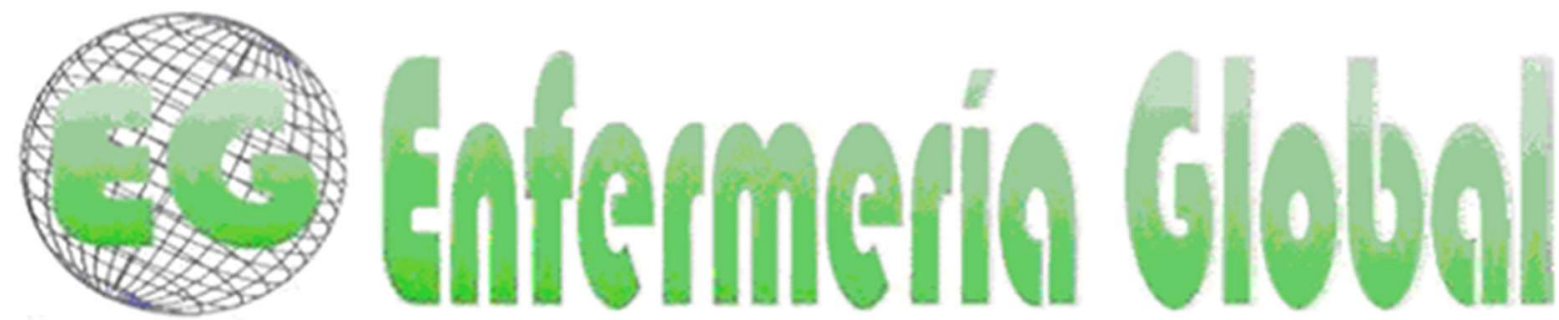

\title{
ORIGINALES
}

\section{Diferentes actitudes hacia la sexualidad entre estudiantes de Enfermería de primero y cuarto curso}

Sexuality education: Different attitudes among incoming and outgoing Nursing undergraduates

\author{
Laura Alonso-Martínez ${ }^{1}$ \\ Davinia Heras-Sevilla ${ }^{1}$ \\ Lucy Charilaou ${ }^{2}$ \\ Jesús Puente-Alcaraz ${ }^{3}$ \\ María Fernández-Hawrylak ${ }^{1}$
}

${ }^{1}$ Facultad de Educación, Departamento de Ciencias de la Educación, Área de Didáctica y Organización Escolar. Universidad de Burgos. Burgos. España. lamartinez@ubu.es

${ }^{2}$ Human Resources. South Tyneside y Sunderland National Health System (NHS) Foundation Trust of the UK.

${ }^{3}$ Facultad de Ciencias de la Salud, Departamento de Ciencias de la Salud, Área de Enfermería. Universidad de Burgos. Burgos. España.

\section{https://doi.org/10.6018/eglobal.474821}

Recibido: $29 / 03 / 2021$

Aceptado: $3 / 07 / 2021$

\section{RESUMEN:}

Objetivo: Evaluar si las actitudes hacia la sexualidad adquiridas por los alumnos durante el Grado en Enfermería son suficientes para su desempeño profesional.

Métodos: Se trata de un estudio descriptivo y transversal basado en una encuesta compuesta por escalas estandarizadas que comparó las predisposiciones sexuales entre 101 alumnos de primer curso con 86 de cuarto. Los 187 estudiantes de enfermería, 24 hombres y 163 mujeres tenían entre 18 y 60 años $\left(M_{\text {edad }}=21.21, D T=5.48\right)$. El instrumento utilizado para su evaluación fue un cuestionario anónimo que estaba constituido por preguntas sociodemográficas y las dos siguientes escalas, la Escala de Actitudes hacia la Sexualidad (ATSS-28) y la Escala de Doble Estándar (DSS).

Resultados: Los resultados obtenidos estuvieron cera de ser significativos por curso en la ATSS, $U=3625.50, z=-1.95, p=.052$ y significativos en la DSS, $U=3560.50, z=-2.13, p=.034$. Estos hallazgos indicaron que los alumnos de cuarto tuvieron actitudes más positivas hacia la sexualidad y una menor adherencia a los roles de género. Además, se obtuvo una correlación negativa moderada, $r_{s}=-.307$, $p=.001$, entre la ATSS y la DSS que mostraba asociación entre las actitudes positivas y la menor adhesión a roles de género. Estos resultados sugieren que la formación enfermera tuvo un impacto positivo en sus actitudes.

Conclusión: La enfermería está involucrada de manera central en el cuidado de los pacientes y sus familias. Sus actitudes hacia la sexualidad son importantes en términos de comodidad del paciente, accesibilidad y aceptabilidad de su atención.

Palabras Clave: Actitud del Personal de Salud; Educación; Enfermeras y Enfermeros; Identidad de Género, Sexualidad. 


\begin{abstract}
:
Objective: To evaluate whether attitudes towards sexuality acquired by students during a Spanish nursing degree are sufficient for their performance in professional practice.

Methods: This is a descriptive, cross-sectional, and quantitative study based on a self-completion survey composed of standardized scales that compare sexual attitudes among 101 first-year students with 86 fourth-year students. The 187 nursing students, 24 men and 163 women were between 18 and 60 years old $\left(M_{\mathrm{age}}=21.21, S D=5.48\right)$. The evaluative instrument was an anonymous questionnaire that consisted of sociodemographic questions and two standardized scales: Attitudes Towards Sexuality Scale (ATSS-28) and Double Standard Scale (DSS).

Results: Comparison between cohorts using the Mann-Whitney $U$ test proved to be close to being significant in the ATSS, $U=3625.50, z=-1.95, p=.052$ and significant in DSS, $U=3560.50, z=-2.13$, $p=.034$. These findings indicate that fourth-year students have more positive attitudes towards sexuality and less rigid adherence to gender roles. In addition, a medium negative correlation, $r_{s}=-.307, p=.001$, between ATSS and DSS was obtained, showing a positive association between attitudes and less adherence to gender roles. These results suggest that nursing degree training had a positive impact on sexual attitudes.

Conclusion: Healthcare professionals are centrally involved in the care of patients and families. Nurses' attitudes towards sexuality are important in terms of patient comfort and the accessibility and acceptability of care. Implementation of training in sexuality has a positive effect on nursing care and favours the establishment of global health strategies.
\end{abstract}

Descriptors: Attitude of Health Personnel; Education; Gender identity; Nurses; Sexuality.

\title{
INTRODUCCIÓN
}

La sexualidad es un aspecto inherente del ser humano e incluye la identidad de género, la orientación sexual, la afectividad, las relaciones afectivas, el placer y la reproducción (1). El desarrollo sexual de los seres humanos está ligado a las experiencias de vida y se manifiesta a través de actitudes, comportamientos, creencias, roles y deseos ${ }^{(2)}$. Este desarrollo ocurre de manera continuada y se integra durante los diferentes procesos y etapas de la vida, desde el nacimiento hasta la muerte. Por tanto, las experiencias sexuales tendrán una gran influencia en el bienestar y la salud de cada individuo ${ }^{(3)}$. Según la Organización Mundial de la Salud (OMS) en 2021, la salud sexual se define como: "un estado de bienestar físico, mental y social en relación con la sexualidad. Requiere un enfoque positivo y respetuoso de la sexualidad $y$ de las relaciones sexuales, así como la posibilidad de tener experiencias sexuales placenteras y seguras, libres de toda coacción, discriminación y violencia" (4).

Esta definición enfatiza el hecho de que la salud sexual es más que la ausencia de enfermedad, es un constructo complejo formado por dimensiones biológicas, psicológicas y socioculturales y se encuentra influenciado por factores económicos e históricos ${ }^{(1)}$.

En lo que respecta a la salud sexual, actualmente hay un millón de personas en el mundo con una privación de su estado de bienestar debido a la propagación de Infecciones de Transmisión Sexual (ITS) ${ }^{(5)}$. La OMS ha estimado que las estrategias llevadas a cabo para prevenir la propagación de las ITS son insuficientes ya que hay un millón de personas que las adquieren todos los días. Además, 376 millones de personas viven con clamidiasis, gonorrea, sífilis o tricomoniasis, 500 millones de personas tienen el virus del herpes simple, 290 millones de mujeres están infectadas con el virus del papiloma humano y 38 millones de personas viven con el virus de la inmunodeficiencia humana (VIH). ${ }^{(1,4,5)}$. Por consiguiente, la sociedad actual no puede seguir permitiendo que los grupos vulnerables sigan presentando las tasas más altas 
de estas enfermedades y que carezcan de medios adecuados para acceder a los servicios de salud. Por tanto, la OMS y la Organización de las Naciones Unidas para la Educación, la Ciencia y la Cultura (UNESCO) han respondido conjuntamente a este problema orientando sus esfuerzos hacia el establecimiento de programas globales de investigación sobre infecciones sexuales (6). Sin embargo, las estrategias preventivas y de promoción de la salud de las ITS no están siendo suficientes, debido a la complejidad de los comportamientos sexuales humanos ${ }^{(5,6)}$ y para mejorar estas intervenciones es necesario invertir en programas de salud, investigación, educación y actividades que brinden una atención eficiente y holística a nivel mundial, limitando las desigualdades entre países ${ }^{(6)}$. La responsabilidad de los programas preventivos de la salud está actualmente en manos de los profesionales educativos y sanitarios, pero para lograr un cambio significativo, se necesita la participación política y la financiación desde los gobiernos de cada país ${ }^{(7)}$.

La educación para la salud sexual debe abordar, normalizar e integrar el estudio de la sexualidad en el currículo, sin enfocarse únicamente en la prevención de enfermedades (8). Esta transformación es necesaria para eliminar las visiones regresivas y prohibitivas de la educación sexual y especialmente de la sexualidad femenina y de las minorías sexuales ${ }^{(9)}$. En los últimos años, la provisión de educación integral en sexualidad se ha vuelto importante, ya que es necesario subrayar e incorporar aspectos relacionados con las actitudes y comportamientos sexuales con otras variables como el género, el respeto a los derechos humanos, y el apego ${ }^{(8,9)}$. Por esta razón, esta formación está diseñada para buscar alcanzar la igualdad entre la diversidad sexual y genérica, garantizar una comunicación interpersonal y social de tolerancia, respetar los derechos de las minorías étnicas y comprender las relaciones de apego ${ }^{(3)}$.

Por tanto, las intervenciones en educación para la salud sexual deben enfocarse en evitar actitudes negativas hacia la sexualidad (erotofobias) y fomentar las actitudes erotofílicas ${ }^{(10,11)}$. De esta manera se avanzará en la reducción de los prejuicios sexistas y la discriminación hacia la comunidad LGTBIQ+ (Lesbiana, Gay, Bisexual, Transgénero, Intersexual, Queer y + , lo que indica la inclusión de una gama más amplia de identidades no representadas en esta abreviatura) ${ }^{(7)}$. La metodología de enseñanza más sustentada científicamente es la presente propuesta de educación sexual integral, que se basa en un modelo biográfico y profesional aceptado mundialmente como el referente para comprender la sexualidad humana. Este método incluye una atención integral al desarrollo de predisposiciones afectivas y a saber cómo abordar las conductas sexuales de las personas a lo largo de la vida (12). Consecuentemente, los profesionales de la salud deberían recibir una formación cualificada que incluya este enfoque y contribuya a generar un cambio significativo en la comprensión y en la adquisición de competencias interdisciplinarias de esta área de conocimientos ${ }^{(8)}$.

\section{Sexualidad en la formación de profesionales de la salud y competencias de enfermería}

La formación de los profesionales de la salud debe abordarse desde un plan de estudios que integre, normalice, comprenda y analice la sexualidad y a su vez sus experiencias y creencias (13). El alumnado universitario de últimos cursos y las personas con formación previa en salud pública muestran actitudes y comportamientos más positivos hacia la sexualidad que el resto de los estudiantes de 
pregrado. Estos hallazgos están relacionados con la idea de maduración de pensamientos sobre la sexualidad y con la formación continuada (14). Sin embargo, el aprendizaje inicial del profesorado en las escuelas y universidades es insuficiente en cuanto a la falta de integración de la sexualidad en sus asignaturas $(14,15)$. La educación que los estudiantes de grados sanitarios han recibido de los docentes se ha centrado principalmente en la prevención de ITS ${ }^{(8)}$. Por ello, es fundamental cambiar esta situación y obligar a los profesores a recibir una formación de calidad en este dominio ${ }^{(15)}$.

Por un lado, investigaciones previas han hallado que los estudiantes universitarios aprenden a abordar la sexualidad de manera desigual, ya que los planes de estudio y la formación docente son diferentes según la carrera elegida ${ }^{(9)}$. Otro de los grandes inconvenientes es que los profesores universitarios manifiestan temor a las reacciones que puedan derivarse de abordar cuestiones relacionadas con la sexualidad y prefieren no tratarlas en el aula, justificando que el alumnado ya ha sido formado en niveles educativos inferiores. Además, los docentes varones tienen una disposición sexual más negativa y regresiva de la sexualidad presentando mayores actitudes erotofóbicas que las profesoras. Esta diferencia de predisposiciones por sexo se relacionaba con las preconcepciones conservacionistas de la adhesión a los roles de género masculinos ${ }^{(14)}$. Curiosamente el número de profesores varones es mayor en las universidades que el de mujeres. Por otro lado, los docentes exponen que estarían dispuestos a participar si reciben formación previa para afrontar esta educación y si la institución les va a respaldar si siguen los protocolos educativos que se establezcan y de esta manera contribuir a generar un cambio positivo que reduce las actitudes y conductas sexistas en la universidad (9-11).

Los profesionales de la salud instruidos y competentes en sexualidad contribuyen a reducir las conductas de riesgo y los prejuicios sexuales, garantizando los derechos sexuales humanos el respeto a las minorías sexuales (13). Por consiguiente, la educación sexual integral debería ser incorporada en los planes de estudio de los egresados y graduados de enfermería con la intención de solucionar la formación insuficiente en esta especialidad y crear equipos sanitarios interdisciplinares que sea capaz de aproximarse y resolver dilemas de índole sexual (13). La solución para mejorar la formación profesional de enfermería en sexualidad radica en una educación interdisciplinaria basada en el cuidado global a través de metodologías activas y científicas que velen por el desarrollo de un currículum en constante actualización al contexto vigente $(8,13)$.

Por otro lado, debemos señalar que las competencias del título de Grado en Enfermería español analizadas en esta investigación cumplen con los principios de calidad de las profesiones establecidos a nivel nacional por el Consejo Internacional de Enfermería y las competencias transversales genéricas del Proyecto Tuning $(16,17)$. Las asignaturas del Grado en Enfermería cumplen con los principios de calidad de las profesiones establecidas en la Agencia Nacional de Evaluación de la Calidad y Acreditación de España (ANECA) y en otros organismos internacionales europeos a través de la Declaración de Bolonia (16). A pesar de ello, estas competencias son generalistas en educación para la salud y no mencionan explícitamente la educación sexual. De esta manera, no habilitan a los estudiantes a adquirir las capacidades que les permitan abordar integralmente la sexualidad. Los contenidos en sexualidad en los Grados de Enfermería españoles se encuentran divididos en bloques temáticos sin hacer explícitos las conexiones transversales que debería haber entre estos 
constructos $(15,18)$. Concretamente, en el grado evaluado de la Universidad Complutense de Madrid, los métodos anticonceptivos se enseñan en la asignatura de Enfermería Materna y las disposiciones y comportamientos sexuales se enseñan en Sociología sin ninguna conexión entre ellos ${ }^{(14,18)}$.

Según Dawson et al. y de Vries et al., no existe una evaluación suficientemente integral de la sexualidad en el currículo de enfermería, y los autores destacan en particular la falta de evaluaciones de las competencias de los estudiantes y los profesores ${ }^{(13,15)}$. Además, los alumnos de ciencias de la salud consideran que estos planes están desactualizados y no se sienten preparados para abordar la diversidad sexual en su área profesional, demandando que sean incluidos en sus planes de estudio (15). Por lo tanto, es fundamental formar enfermeras que puedan contribuir a un cambio disciplinario positivo hacia la sexualidad a través de sus roles laborales ${ }^{(1,13)}$.

Este estudio tiene como objetivo principal evaluar las actitudes hacia la sexualidad y la adhesión a roles sexuales, comparando las predisposiciones manifestadas por los estudiantes de primer y último curso del Grado de Enfermería. Como propósitos secundarios, esta investigación también tiene como objetivo valorar si las actitudes difieren por género, edad y otras variables sociodemográficas de interés analizadas en el estudio. Las hipótesis planteadas en esta investigación fueron que los estudiantes de enfermería de primero y último curso sugerirán de manera similar que adquirieron una mejora insuficiente en sus competencias profesionales relacionadas con el abordaje de la sexualidad humana. Se asume que el alumnado, a pesar de haber recibido educación sexual formal en la prevención de ITS, declarará una elevada tasa de conductas sexuales de riesgo. Además, se hipotetizó que las mujeres, los estudiantes con formación previa y de mayor edad como los alumnos de cuarto tendrán actitudes más positivas hacia la sexualidad y menor adhesión a los roles de género que el resto de los participantes. Finalmente, en relación con la literatura previamente analizada se asume que las actitudes positivas o liberales hacia la sexualidad correlacionarán negativamente con el sostenimiento de un doble rol sexual.

\section{MÉTODOS}

\section{Tipo de estudio y participantes}

Se ha propuesto un diseño de estudio cuantitativo no experimental de carácter transversal y descriptivo, dada la relevancia de esta modalidad para explorar hechos, circunstancias o fenómenos y examinar las relaciones entre los factores. Las variables sociodemográficas son género, edad, cohorte y formación previa, y las variables dependientes fueron los resultados de las dos escalas y las preguntas sobre conductas sexuales de riesgo y educación sexual. Para lograr el objetivo propuesto se ha realizado un estudio comparativo-correlacional mediante la utilización de un cuestionario. Se realizó un muestreo incidental no probabilístico, que utilizó la disponibilidad de estudiantes matriculados en el Grado en Enfermería. La muestra está compuesta por 187 estudiantes, entre 18 y 60 años $(M=21.21, S D=5.48)$ matriculados en enfermería en la Universidad Complutense de Madrid (UCM, España) durante el curso 2017-2018. Los estudiantes universitarios fueron reclutados voluntariamente para participar en el estudio a través de métodos de muestreo 
oportunistas. Los criterios de inclusión en la investigación fueron que todos los estudiantes fueran alumnos de enfermería de primer y último curso de la UCM, y los criterios de exclusión fueron aquellos que no quisieron participar y estudiantes menores de edad.

\section{Herramientas de medición}

El interés principal de este estudio fue evaluar la educación sexual, las conductas sexuales de riesgo, la adhesión a roles sexuales de género y las actitudes negativas y positivas hacia la sexualidad. Así, el instrumento de estudio se dividió en 3 apartados: en primer lugar, el apartado A contenía 6 preguntas autoelaboradas sobre datos personales, como fueron las variables edad, género, orientación sexual, curso universitario, formación previa, opiniones sobre la educación sexual recibida y realización de conductas de riesgo; en segundo lugar, la sección B contenía las 2 escalas estandarizadas antes mencionadas y, por último, la sección C contenía 1 pregunta autoelaborada en la que se consultaba los comentarios y las sugerencias. Este cuestionario también contenía la pregunta de consentimiento informado.

La versión en español de la Escala de Actitudes hacia la Sexualidad (ATSS) de Fisher y Hall (19) utilizada en esta investigación fue la adaptación validada y ampliada por Diéguez et al. ${ }^{(20)}$. Esta escala se utiliza para evaluar el grado de liberalismo sexual y las actitudes erotofílicas y erotofóbicas hacia sexualidad a través de sus 28 ítems. Las respuestas se estructuran en torno a una escala de Likert de 5 puntos $(1=$ Totalmente en desacuerdo a 5 = Totalmente de acuerdo). Los ítems $1,4,5,8,9,12,14,16,17$, $18,21,22,27$ y 28 fueron redactados negativamente, por lo que su valor debe invertirse antes del análisis. La evaluación consiste en valorar los rangos de la suma de la puntuación de los ítems de la escala. La puntuación varía de 28 a 140 puntos. Los valores más bajos indican un menor grado de liberalismo y actitudes más negativas hacia la sexualidad. Fisher y Hall (19) obtuvieron un Alfa de Cronbach aceptable: $\alpha=.76$ para el rango de 12 a 14 años. En nuestra investigación se obtuvo una confiabilidad buena con un $\alpha=.81$.

La versión en español de la Double Standard Scale (DSS) de Caron et al. ${ }^{(21)}$ utilizada en esta investigación fue la adaptación validada por Sierra, et al. (22). Esta escala se utiliza para evaluar las actitudes hacia el grado de adherencia al doble estándar sexual, a través de sus 10 ítems. Las respuestas se estructuran en torno a una escala de Likert de 5 puntos ( 1 = Totalmente en desacuerdo a 5 = Totalmente de acuerdo). El elemento 8 está redactado negativamente, por lo que su valor debe invertirse antes del análisis. La evaluación consiste en valorar los rangos de la suma de la puntuación de los ítems de la escala que varían de 10 a 50 puntos. Los valores más altos indican una alta adherencia al doble estándar sexual. Caron et al. (21) obtuvieron un Alfa de Cronbach aceptable de .72 en adultos. En nuestro estudio se obtuvo una fiabilidad aceptable con un $\alpha=.71$.

\section{Recopilación de datos}

Este estudio fue aprobado por el comité de ética de la UCM. Todos los alumnos participaron voluntariamente en el estudio al aceptar el formulario de consentimiento. La recogida de datos se llevó a cabo en Madrid, España, en los meses de marzo y junio (para estudiantes de primer curso y de último) del año 2018. Se llevó a cabo en la Universidad y el Hospital Clínico San Carlos (Madrid). Los participantes se 
reunieron en las aulas y se les entregaron los cuestionarios para completarlos. El instrumento se completaba en unos 25 minutos por grupo. Los cuestionarios fueron administrados por el investigador en persona, en formato online y a través de investigadores colaboradores para asegurar el rigor científico. El procedimiento se dividió en 3 partes: revisión de la literatura, elaboración de la encuesta y pilotaje; administración de encuestas; y análisis de resultados.

\section{Análisis de los datos}

Los datos fueron informatizados y analizados a través de IBM SPSS 24. Las variables cuantitativas se representaron en media y desviación estándar (DT) y las categóricas en números y porcentajes. Los datos se exploraron antes del análisis para examinar la normalidad de los resultados característicos de la muestra. Se indicó una distribución no normal en las puntuaciones medias de las escalas mediante la realización de la prueba de Kolmogorov-Smirnov. Este análisis mostró para el ATSS, $D(187)=.140, p=.001$ y para el DSS, $D(187)=.084, p=.002$. Por tanto, las escalas se analizaron en comparación con las variables sociodemográficas de nuestro estudio mediante estadística no paramétrica. En primer lugar, se realizó una prueba $U$ de Mann-Whitney para muestras independientes con el fin de comparar los rangos medios de las puntuaciones de la escala entre dos grupos, como serían las variables de género, cohorte y entrenamiento previo. Por último, se realizó el coeficiente de correlación de rangos de Spearman para determinar si había una relación estadísticamente significativa entre la puntuación de las escalas y la edad. El nivel de significancia en el análisis de inferencias estadísticas se basó en valores de $p<.05$.

\section{RESULTADOS}

\section{Estadística descriptiva}

El perfil de los participantes de esta muestra se describirá a continuación en la Tabla 1 según las variables de género, cohorte académico y formación previa.

Tabla 1. Características de la muestra

\begin{tabular}{|c|c|c|c|c|}
\hline \multirow[t]{2}{*}{ Variables } & \multicolumn{2}{|c|}{ Cohorte académico ( $n$ y $\%)$} & \multirow[t]{2}{*}{$N$} & \multirow[t]{2}{*}{$\%$} \\
\hline & $\begin{array}{c}\text { Alumnos de } \\
\text { primero }\end{array}$ & $\begin{array}{l}\text { Alumnos de } \\
\text { cuarto }\end{array}$ & & \\
\hline \multicolumn{5}{|l|}{ Género } \\
\hline Mujer & $92(91.08 \%)$ & $71(82.55 \%)$ & 163 & 87.16 \\
\hline Hombre & $9(8.91 \%)$ & $15(17.44 \%)$ & 24 & 12.83 \\
\hline $\begin{array}{l}\text { Otra formación en educación superior } \\
\text { relaciona con Ciencias de la Salud }\end{array}$ & & & 10 & 5.34 \\
\hline Poseen otra formación & $14(13.86 \%)$ & $15(17.44 \%)$ & 29 & 15.50 \\
\hline No poseen otra formación & $87(86.13 \%)$ & $71(82.55 \%)$ & 158 & 84.49 \\
\hline Total & $101(54.01 \%)$ & $86(45.98 \%)$ & 187 & 100 \\
\hline
\end{tabular}


A continuación, en la Tabla 2 se explican las opiniones sobre educación sexual y los comportamientos de comunicación sexual mostrados por los estudiantes del Grado de Enfermería por cohortes. En esta tabla se muestra en línea con la hipótesis 1, el $95,2 \%$ de los estudiantes considera que la información recibida en esta área no ha sido suficiente, coincidiendo también que el $92,5 \%$ de los alumnos piensa que es importante recibir esta formación para el desarrollo de sus competencias profesionales. Curiosamente, los participantes indicaron que han recibido una educación en sexualidad más informal que basada en la evidencia científica, con un alto grado de similitud entre los grupos de primero y segundo. Por otro lado, en línea con la hipótesis 2, los estudiantes de pregrado presentaron altos porcentajes en la falta de conciencia en la comunicación de conductas sexuales de riesgo. El $42 \%$ de los estudiantes siempre usó condón en las relaciones sexuales, mientras que el $58 \%$ había tenido relaciones sexuales sin protección. Además, de estos solo el $5 \%$ de los estudiantes se sometió a una prueba de ITS antes de tener relaciones sexuales sin protección con sus parejas y a su vez únicamente el $15 \%$ realizó una prueba serológica cuando tuvieron relaciones sexuales sin protección con una nueva pareja.

\section{Tabla 2. Educación sexual y conductas de riesgo del alumnado}

\begin{tabular}{|c|c|c|c|c|}
\hline \multirow[t]{2}{*}{ Variables } & \multicolumn{3}{|c|}{ Cohorte académico $(n$ y $\%) N$} & \multirow[t]{2}{*}{$\%$} \\
\hline & Primer curso & Cuarto curso & & \\
\hline \multicolumn{5}{|l|}{ Formación en Educación para la Salud Sexual } \\
\hline Recibida & $78(77.22 \%)$ & $70(81.39 \%)$ & 148 & 79.14 \\
\hline No recibida & $23(22.77 \%)$ & $16(18.60 \%)$ & 39 & 20.85 \\
\hline \multicolumn{5}{|l|}{$\begin{array}{l}\text { Educación sexual y formal recibida en el Grado en } \\
\text { Enfermería }\end{array}$} \\
\hline Estudiantes que manifestaron haberla recibido & $4(3.96 \%)$ & $7(8.13 \%)$ & 11 & 5.88 \\
\hline Estudiantes que manifestaron no recibirla. & $97(96.03 \%)$ & $79(91.86 \%)$ & 176 & 94.11 \\
\hline \multicolumn{5}{|l|}{ Calidad de la educación sexual recibida } \\
\hline Los estudiantes lo consideraron buena o extremadamente buena & $6(5.94 \%)$ & $3(3.48 \%)$ & 9 & 4.81 \\
\hline Los estudiantes lo consideraron malo o extremadamente malo & $95(94.05 \%)$ & $83(96.51 \%)$ & 178 & 95.18 \\
\hline \multicolumn{5}{|l|}{ Utilidad de la formación en sexualidad para su profesión } \\
\hline Los estudiantes lo consideraron útil o extremadamente útil & $89(88.11 \%)$ & $84(97.67 \%)$ & 173 & 92.51 \\
\hline Los estudiantes lo consideraron poco útil o nada útil. & $12(11.88 \%)$ & $1(1.16 \%)$ & 13 & 6.95 \\
\hline Estudiantes que decidieron no responder & 0 & $1(1.16 \%)$ & 1 & 0.53 \\
\hline \multicolumn{5}{|l|}{ Participación voluntaria en tareas formativas en sexualidad } \\
\hline Estudiantes que lo estaban realizando & $7(6.93 \%)$ & $6(6.97 \%)$ & 13 & 6.95 \\
\hline Estudiantes que no lo estaban realizando. & $94(93.06 \%)$ & $80(93.02 \%)$ & 174 & 93.54 \\
\hline \multicolumn{5}{|l|}{ Formación a través de recursos evidenciados científicamente } \\
\hline Estudiantes que manifestaron que lo han recibido & $40(39.60 \%)$ & $25(29.06 \%)$ & 65 & 34.75 \\
\hline Estudiantes que manifestaron que no lo han recibido. & $60(59.4 \%)$ & $61(70.93 \%)$ & 121 & 64.70 \\
\hline Estudiantes que decidieron no responder & $1(0.99 \%)$ & 0 & 1 & 0.53 \\
\hline \multicolumn{5}{|l|}{ Educación informal: familia, amigos e Internet (no científica) } \\
\hline Estudiantes que manifestaron que lo han recibido & $79(78.21 \%)$ & $65(75.58 \%)$ & 144 & 78.60 \\
\hline Estudiantes que manifestaron que no lo han recibido. & $22(21.78 \%)$ & $21(24.41 \%)$ & 43 & 22.99 \\
\hline \multicolumn{5}{|l|}{ Competencias en sexualidad del alumnado } \\
\hline Se consideran competentes o extremadamente competentes & $27(26.73 \%)$ & $41(47.67 \%)$ & 68 & 36.36 \\
\hline Se consideran poco competentes o nada competentes & $74(73.26 \%)$ & $45(52.32 \%)$ & 119 & 63.63 \\
\hline
\end{tabular}




\begin{tabular}{|c|c|c|c|c|}
\hline \multicolumn{5}{|c|}{$\begin{array}{l}\text { Utilizo el condón como método anticonceptivo cuando tengo } \\
\text { relaciones sexuales con una nueva pareja }\end{array}$} \\
\hline Siempre & $37(36.66 \%)$ & $41(47.67 \%)$ & 78 & 41.71 \\
\hline Nunca & $61(60.39 \%)$ & $38(44.18 \%)$ & 99 & 52.94 \\
\hline Decidió no responder & $3(2.97 \%)$ & $7(8.13 \%)$ & 10 & 5.34 \\
\hline \multicolumn{5}{|c|}{$\begin{array}{l}\text { Mi pareja y yo nos hemos hecho una prueba serológica antes } \\
\text { de tener relaciones sexuales sin protección. }\end{array}$} \\
\hline Siempre & $3(2.97 \%)$ & $7(8.13 \%)$ & 10 & 5.34 \\
\hline Nunca & $94(93.06 \%)$ & $72(83.72 \%)$ & 166 & 88.77 \\
\hline Decidió no responder & $4(3.96 \%)$ & $7(8.13 \%)$ & 11 & 5.88 \\
\hline \multicolumn{5}{|c|}{$\begin{array}{l}\text { Me hice una prueba serológica después de haber tenido } \\
\text { relaciones sexuales sin protección con una nueva pareja. }\end{array}$} \\
\hline Siempre & $16(15.84 \%)$ & $12(13.95 \%)$ & 28 & 14.97 \\
\hline Nunca & $82(81.19 \%)$ & $70(81.39 \%)$ & 152 & 81.28 \\
\hline Decidió no responder & $3(2.97 \%)$ & $4(4.65 \%)$ & 7 & 3.74 \\
\hline Total & $101(54.01 \%)$ & $86(45.98 \%)$ & 187 & 100 \\
\hline
\end{tabular}

$n=$ muestra, $\%=$ porcentaje, $N=$ población total

Finalmente, en la Tabla 3 se detallan los resultados descriptivos de la variable edad y de las puntuaciones de la ATSS y la DSS, en la cual se incluyen los datos de la muestra divididos en cohorte académico (primero y cuarto) y total.

Tabla 3. Estadísticos descriptivos por edad y escala

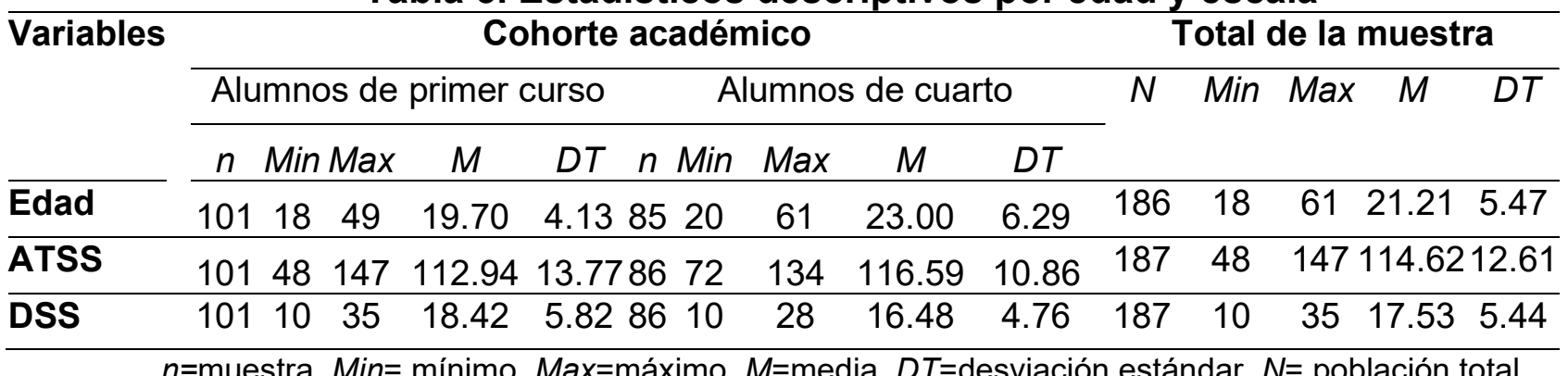

\section{Estadística inferencial}

Se encontró que el análisis de la ATSS de Fisher y Hall (19) estuvo cerca de ser estadísticamente significativo entre las cohortes, en línea con la hipótesis 3 . Fue menor para los estudiantes universitarios de primer curso $\left(M_{\text {rank }}=86.90\right.$, Sum of Ranks $=8776.50, n=101$ ) que para los alumnos de cuarto curso (Mrank $=102.34$, Sum of Ranks = 8801.50, $n=86$ ), $U=3625.50, z=-1.95, p=.052$ (2-tailed). Estas diferencias de rango mostraron una actitud más positiva hacia la sexualidad en los estudiantes de cuarto curso. Sin embargo, contradictoriamente a esta hipótesis, el género y el entrenamiento previo no predijeron la puntuación de la ATSS, ver Tabla 4. 
Tabla 4. Prueba U de Mann-Whitney para ATSS por género y otra formación

\begin{tabular}{|c|c|c|c|c|c|c|}
\hline Variables & $\begin{array}{l}\text { Rango } \\
\text { medio }\end{array}$ & $\begin{array}{l}\text { Sumatorio de } \\
\text { rango }\end{array}$ & $\begin{array}{c}\text { Mann- } \\
\text { Whitney } \\
U\end{array}$ & $\begin{array}{l}\text { Wilcoxon } \\
\quad W\end{array}$ & $z$ & $p$ \\
\hline \multicolumn{7}{|l|}{ Género $(n=187)$} \\
\hline Hombre $(n=24)$ & 88.94 & 2134.50 & 1834.50 & 2134.50 & -.491 & .623 \\
\hline Mujer $(n=163)$ & 94.75 & 15443.50 & & & & \\
\hline \multicolumn{7}{|l|}{ Otra formación $(n=187$} \\
\hline Posee otra formación $(n=29)$ & 94.47 & 2797.50 & 2219.50 & 14780.50 & -.267 & .789 \\
\hline No posee otra formación $(n=158)$ & 93.55 & 14780.50 & & & & \\
\hline
\end{tabular}

Además, en contradicción con esta misma hipótesis, la correlación de rango positiva por edad y puntajes de la ATSS, $r_{s}=.035, p=.634$ no fue significativa.

Sin embargo, de acuerdo con la hipótesis 4 , el análisis de la DSS de Caron, et al.(21) se encontraron diferencias significativas entre cohortes. Fue significativamente más alto para los estudiantes universitarios de primer curso $\left(M_{\text {rank }}=101.75\right.$, Sum of Ranks $=10276.50, n=101)$ que para los alumnos de cuarto curso $\left(M_{\text {rank }}=84.90\right.$, Sum of Ranks $=7301.50, n=86$ ), $U=3560.50, \mathrm{z}=-2.126, p=.034$ (2-tailed). Estas diferencias de rango mostraron una menor adherencia a los roles de género en los estudiantes de cuarto curso. Sin embargo, contrariamente a esta hipótesis y como en la escala anterior, el género y la formación previa no predijeron la puntuación de la DSS, ver Tabla 5.

Tabla 5. Prueba U de Mann-Whitney para DSS por género y otra formación

\begin{tabular}{lcccccc}
\hline Variables & $\begin{array}{c}\text { Rango } \\
\text { medio }\end{array}$ & $\begin{array}{c}\text { Sumatorio } \\
\text { de rango }\end{array}$ & $\begin{array}{c}\text { Mann- } \\
\text { Whitney } \boldsymbol{U}\end{array}$ & $\begin{array}{c}\text { Wilcoxon } \\
\boldsymbol{W}\end{array}$ & $\mathbf{z}$ & $\boldsymbol{p}$ \\
\hline Género $(n=187)$ & & & & & & \\
$\quad$ Hombre $(n=24)$ & 97.23 & 2333.50 & 1878.50 & 15244.50 & -.314 & .754 \\
$\quad$ Mujer $(n=163)$ & 93.52 & 15244.50 & & & & \\
\hline$\quad$ Otra formación $(n=187$ & & & & & & \\
$\quad$ Posee otra formación $(n=29)$ & 96.86 & 2809.00 & 2208.00 & 14769.00 & -.310 & .756 \\
$\quad$ No posee otra formación $(n=158)$ & 93.47 & 14769.00 & & & & \\
\hline
\end{tabular}

Además, no se encontró una correlación de rango negativa por edad y puntajes de la DSS, $r_{s}=-.054, p=.464$. Finalmente, en línea con la hipótesis 5 , se descubrió una correlación negativa media entre los rangos de la ATSS y los puntajes de la DSS, $r_{s}=-$ $.307, p=.001$. Este hallazgo indica que una menor adherencia a los roles de género (DSS) está relacionada con actitudes más erotofílicas hacia la sexualidad y mayores predisposiciones de liberalismo sexual.

\section{DISCUSIÓN}

Esta investigación se llevó a cabo para evaluar la educación en sexualidad recibida, las conductas sexuales de riesgo, las actitudes generales hacia la sexualidad y la 
adhesión a los roles sexuales en el Grado en Enfermería, comparando las opiniones, predisposiciones y comportamientos de los estudiantes universitarios de primero y último curso. Los hallazgos han alcanzado el objetivo y han apoyado las hipótesis, aunque no se han encontraron diferencias significativas por género y otra formación previa.

El $77 \%$ de los estudiantes de enfermería manifestaron que su entrenamiento en educación para la salud sexual se ha transmitido mayoritariamente de forma informal y no científica (familia, amigos o Internet). Por tanto, esta educación del currículum oculto está contribuyendo al mantenimiento de normas, mitos y falsas creencias heteronormativas y heteropatriarcales que no se encuentran fundamentadas en la evidencia científica (3). La mayor parte de la educación regulada en el currículum se imparte desde un modelo biológico, sin comprender las implicaciones socioculturales y psicológicas (15). Para solucionar esta problemática, es necesario legislar los contenidos y competencias en los planes de estudio de Enfermería y así contribuir a que los docentes enfermeros modifiquen sus guías docentes y eviten la transmisión de un currículo desadaptado a las problemáticas vigentes ${ }^{(13)}$. La formación de los alumnos de enfermería en sexualidad basada en la evidencia científica en esta temática les permitirá convertirse en profesionales críticos y competentes para abordar las situaciones sexuales de los usurarios a los que cuidan. Estas enseñanzas también prepararán a estos estudiantes de la salud para gestionar estrategias preventivas, de promoción y de educación para la salud desde un enfoque psicoafectivo y psicosexual integral (2).

En esta investigación, solo el $7 \%$ de los alumnos se involucró en actividades educativas en sexualidad, por lo que son un porcentaje minoritario de la muestra. Sin embargo, los participantes que más se involucraron en estas actividades fueron lo que tenían las actitudes más negativas hacia la sexualidad en comparación con el promedio de los otros estudiantes. Por otro lado, en relación con la hipótesis 1, con la edad y los factores de formación profesional, los estudiantes de cuarto año se consideraron más capaces de educar a la población en sexualidad que los estudiantes de primer año (11). No obstante, el porcentaje de estudiantes que habían adquirido comportamientos sexuales saludables a través de sus propias experiencias y conocimientos estaban muy por debajo del promedio y en algunas premisas han sido inferiores al 5\%, demostrando un comportamiento de alto riesgo sexual a pesar de sus conocimientos sanitarios. Esto implica que la formación en el módulo de la sexualidad biológica centrada en las ITS no ha generado un cambio significativo en la reducción de sus conductas sexuales de riesgo ${ }^{(15)}$, sin apenas diferencias por curso.

Las actitudes y creencias hacia el liberalismo sexual en esta investigación se obtuvieron mediante el análisis de la ATSS de Fisher y Hall (19). Los estudiantes presentaron una puntuación promedio de 114,72 que indica valores positivos hacia el liberalismo y las actitudes sexuales. En línea con la hipótesis 3, se encontraron diferencias significativas en los puntajes de la ATSS, reflejando que los estudiantes de primer curso poseen menor grado de liberalismo y mayores actitudes erotofóbicas que los alumnos de cuarto año. No se obtuvieron diferencias en función de otros niveles de formación previa la grado. Al comparar esta investigación con la de Díaz et al. (23), sus hallazgos en la ATSS indicaron actitudes más negativas hacia la sexualidad en una muestra de adultos discapacitados y no discapacitados $(M=86.49, D T=16.97$, $n=729$ ) con edades comprendidas entre los 19 y los 55 años que las obtenidos en nuestro estudio. Es reconocido mundialmente que la edad y los factores 
socioculturales tienen un gran impacto en la predisposición sexual. Gómez-Zapiain et al. (9) afirman que la edad influye en los comportamientos y actitudes sexuales y contribuye a disminuir los estereotipos sexuales. Además, investigaciones previas en estudiantes de pregrado de similar edad de Diéguez, et al. ${ }^{(23)}(M=111,7, D T=11,82$, $n=5614)$, con una media de edad de 20.7 años y un mayor porcentaje de mujeres $(59,3 \%)$ y Diéguez, et al. (20) $(M=111.64, D T=12.63, n=2006)$ con participantes de entre 17 y 52 años, también obtuvieron una puntuación media inferior a la de nuestro estudio. Al igual que en nuestro estudio, Diéguez, et al. ${ }^{(20)}$ tampoco encontraron diferencias significativas por género, pero si obtuvieron diferencias significativas más positiva en los estudiantes de cuarto frente a los de primer curso en participantes con rangos de edad similares a los nuestros.

Según la hipótesis 4, se encontraron diferencias significativas en los puntajes de la DSS por cohorte que reflejan una mayor adherencia al rol sexual en los estudiantes de primer año en comparación con los estudiantes de cuarto curso, pero tampoco se encontraron diferencias entre el género y otros niveles de formación. Sin embargo, en los estudios de Caron et al (21) llevados a cabo con alumnos de primer curso de grado $(M=19.1, \quad D T=5.3, n=330)$, y el de Sierra et al. (22) realizados con alumnos universitarios españoles $(M=17.9, D T=5.6, n=400)$, los hombres presentaron una adherencia más negativa a los roles sexuales que las mujeres. Además, Sierra y Gutiérrez (25) también encontraron mayores adherencias negativas a los roles sexuales en los hombres universitarios $(M=20.69, D T=6.53, n=192)$ que en las mujeres $(M=16.15, D T=5.7, n=223)$, con un rango de edad de 16 a 46 años. No obstante, estos hallazgos muestran que dentro de la variable de género las alumnas de Grado en Enfermería de nuestra muestra $(M=17.5, D T=5.5, n=163)$ tienen una mayor adherencia a los roles sexuales que en los estudios enumerados anteriormente. Sin embargo, en esta investigación la puntuación media total de la DSS ha sido más baja que la del resto de los estudios señalados.

Las diferencias actitudinales obtenidas entre el alumnado de primero y cuarto curso son pequeñas en la DSS y no significativas en la ATSS. Por tanto, estos resultados positivos hallados en relación con las predisposiciones podrían sustentarse en la maduración sexual de los individuos y en la creación de sociedades más igualitarias basadas en el respeto a los derechos humanos que está ayudando a reducir los estereotipos sexuales y la adhesión a los roles de género (26,27). La formación impartida en las carreras de enfermería debería seguir contribuyendo activamente a generar un cambio significativo en las actitudes hacia la sexualidad, debido a que los roles de estos futuros profesionales de la salud permiten que en su desempeño laboral sean educadores cualificados tanto a través de la enseñanza reglada como informal (28).

Las fortalezas del estudio es que la investigación está basada en las evidencias científicas para medir la sexualidad desde un enfoque cuantitativo, aumentando la información de estas escalas estandarizadas. Este estudio contribuye a comprender que las actitudes hacia la sexualidad están vinculadas con la formación, el interés personal y que están altamente condicionadas por las normas socioculturales ${ }^{(29)}$. Sin embargo, se debe considerar que los cambios positivos producidos hacia las predisposiciones sexuales también pueden estar asociados con la madurez sexual y la implementación de legislaciones que promuevan el respeto de los derechos humanos ${ }^{(30)}$. Por otro lado, a pesar de contar con una formación significativa en el control de las ITS, es aquí donde se encontraron más conductas negativas hacia la 
sexualidad, debido a que estos contenidos se impartieron centrándose en métodos biomédicos que no lograron conectar la sexualidad con la afectividad y los cambios sociales relacionado con los comportamientos de riesgo ${ }^{(29,30)}$.

Las limitaciones de este estudio radican en el tamaño de la muestra y la falta de homogeneidad entre hombres y mujeres. Sin embargo, estas diferencias de género pueden deberse al sesgo que existe en las profesiones de la salud, en las que al ser carreras feminizadas hay una mayor presencia de mujeres. Además, este estudio se centró en evaluar la sexualidad heterosexual sin incluir las perspectivas de las minorías sexuales, dando un resultado limitado en relación con la diversidad sexual humana. En estudios futuros, la muestra debe expandirse a diferentes universidades con diferentes contextos educativos (poblaciones pequeñas y/o grandes), comparar entre distintas ramas formativas y añadir más variables sociodemográficas (nivel socioeconómico, orientación sexual e ideologías políticas). Sería beneficioso para estas investigaciones realizar estudios longitudinales a lo largo del Grado en Enfermería que contrasten los hallazgos obtenidos de este estudio transversal. El resultado de la ATSS también debería estar sujeto a más investigaciones, debido a la obtención de un hallazgo que está cerca de ser significativo. Con relación a la metodología sería conveniente combinar enfoques cuantitativos y cualitativos para examinar las actitudes hacia la sexualidad con más detalle, a fin de proponer intervenciones globales de manera eficaz para cambiar las actitudes y comportamientos negativos. Finalmente, se deben llevar a cabo programas y estrategias educativas impartidas por enfermeras que contribuyan a mejorar la educación para la salud sexual de la población desde una perspectiva holística que no se enfoque únicamente en un modelo biomédico.

\section{CONCLUSIÓN}

Los estudiantes de primero y cuarto curso presentaron conductas sexuales de riesgo similares, y las pequeñas diferencias actitudinales entre estos niveles educativos podría estar más relacionadas con variables sociodemográficas y culturales que con las competencias adquiridas en el Grado de Enfermería, requiriendo de una mayor investigación. La educación de los estudiantes se centró en el modelo biológico que no permite que los alumnos adquieran completamente estas competencias y sigue manteniéndose en un currículum no transversal. La formación en sexualidad de los estudiantes es deficiente, informal y se basa en enfoques biomédicos. Se puede considerar insuficiente porque al analizar su comportamiento comunicativo de riesgo, es evidente que no han adquirido una conducta saludable hacia este. Se requiere de una remodelación educativa para mejorar las actitudes hacia la sexualidad y contribuir a generar roles de género equitativos. La formación profesional de enfermería debe incluir la educación en sexualidad desde una perspectiva holística para mejorar la carrera profesional de las enfermeras. Los programas de educación para la salud sexual deben impartirse en edades educativas tempranas para promover la coeducación y la adquisición de conductas sexuales saludables, incluyendo el género, la igualdad social y las conductas erotofílicas, la prevención de las ITS, los problemas de salud mental y otras conductas sexuales de riesgo. Para concluir, es necesario regular el currículo universitario para formar profesionales cualificados en educación para la salud sexual y, por tanto, la enfermería no debe quedarse atrás en esta nueva iniciativa. Las enfermeras deben tener la oportunidad de coordinar equipos interdisciplinarios e influir en las políticas actuales, para garantizar que exista un 
sistema de salud igualitario y eficaz. La carrera ofrece formación integral del ser humano, investigación científica de calidad, y sus diferentes especialidades avalan a la enfermería como profesión fundamental para la construcción de la educación para la salud y por tanto de la educación sexual.

\section{Reconocimiento}

Este estudio contó con el apoyo de la Facultad de Enfermería, Fisioterapia y Podología de la Universidad Complutense de Madrid. Los autores agradecen a los participantes anónimos y a las enfermeras del Hospital Clínico de San Carlos que ayudaron a recolectar la muestra de los alumnos de cuarto curso.

\section{REFERENCIAS}

1. Foucault M. The History of Sexuality: 1: The Will to Knowledge. UK: Penguin; 2020. Available from: https://www.penguin.co.uk/books/238/23884/the-history-ofsexuality--1/9780241385982.html

2. Benton CP. Sexual health attitudes and beliefs among nursing faculty: A correlational study. Nurse Education Today. 2021; 98: 104665. Available from: https://doi.org/10.1016/j.nedt.2020.104665

3. Grozelle RS. Cultural heterosexism and silencing sexual diversity: AnokaHennepin School District. Journal of LGBT Youth. 2017; 14(4): 393-410. Available from: https://doi.org/10.1080/19361653.2017.1365035

4. WHO-World Health Organization. Sexual health and its linkages to reproductive health: an operational approach, Geneva. [Internet]. 2017[cited 2021 March 10]. Available from: https://www.who.int/publications/i/item/978924151288

5. World Health Organization. Sexually transmitted infections (STIs). Ginevra, Switzerland: World Health Organization. [Internet]. 2019 [cited 2020Aug.12]. Available from: https://www.who.int/news-room/fact-sheets/detail/sexually-transmitted-infections(stis)

6. UNESCO United Nations Educational, Scientific and Cultural Organization. International technical guidance on sexuality education: An evidence-informed approach (second revised edition). Paris: UNESCO [Internet]. 2018 [cited 2020Aug.13]. Available from https://unesdoc.unesco.org/ark:/48223/pf0000260770

7. Alonso-Martínez L, Heras-Sevilla D, Fernández-Hawrylak M, Forrest S. English Validation of a Short Scale Designed to Detect Negative Attitudes towards Trans People (EANT). Sustainability [Internet]. 2021;13(7):3760. Available from: http://dx.doi.org/10.3390/su13073760

8. Pavelová L', Archalousová A, Slezáková Z, Zrubcová D, Solgajová A, Spáčilová $Z$, et al. The Need for Nurse Interventions in Sex Education in Adolescents. International Journal of Environmental Research and Public Health [Internet]. 2021; 18(2):492. Available from: http://dx.doi.org/10.3390/ijerph18020492

9. Gómez-Zapiain J, Ortiz M J, Eceiza A. Disposición al riesgo en la actividad sexual adolescente: el papel de los modelos internos de apego, de las relaciones de apego con padres y pares y de las dificultades en la regulación emocional. Anales de Psicología. 2016; $32 \quad$ (3): 899-906. Available from: http://dx.doi.org/10.6018/analesps.32.3.221691

10. Fisher W A, Byrne D, White LA, Kelley K. Erotophobia-erotophilia as a dimension of personality. Journal of Sex Research. 1998; 25(1): 123-151. https://doi.org/10.1080/00224498809551448 
11. Senturk Erenel A, Cicek Ozdemir S. Attitude and Behaviors of Senior Nursing Students in Assessment of Sexual Health. Sexuality and Disability. 2020; 38, 503-514. Available from: https://doi.org/10.1007/s11195-020-09642-3

12. Heras D, Lara F, Fernández-Hawrylak M. Evaluation of the Effects of the Sexual Education Program SOMOS on Sexual Experience and Attitudes of Adolescents Towards Sexuality 322. Revista Psicodidáctica [Internet]. 2016 [cited 2020 Aug 15]; 21(2): 321-37. Available from: www.ehu.eus/revista-psicodidactica

13. Dawson M, Gakumo A, Philips J, Wilson L. Process for Mapping Global Health Competencies in Undergraduate and Graduate Nursing Curricula. Nurse Educator. 2016;41(1):37-40. Available from: Doi: 10.1097/NNE.0000000000000199

14. Gorrotxategi M P, Ozamiz-Etxebarria N, Jiménez-Etxebarria E, y CorneliusWhite $\mathrm{J} \mathrm{H}$. Improvement in gender and transgender knowledge in university students through the Creative Factory methodology. Frontiers in Psychology; 2020: 11, 367. Available from: $\underline{\text { https://doi.org/10.3389/fpsyg. } 2020.00367}$

15. de Vries E, Kathard H, Müller A. Debate: Why should gender-affirming health care be included in health science curricula?. BMC Medical Education. 2020; 20(1):110. Available from: https://doi.org/10.1186/s12909-020-1963-6

16. Consejo Internacional de Enfermeras. La definición de Enfermería. The International Council of Nurses. 2020 [cited 2020 Aug 17]. Available form: https://www.icn.ch/es/politica-de-enfermeria/definiciones

17. Collegiate Organization of Nursing. Proyect Tuning: Tuning Educational Structures in Europe [Internet]. Collegiate Organization of Nursing. 2020 [cited 2020 Aug 18]. Available from: https://www.consejogeneralenfermeria.org/primer-ciclo/perfildel-profesional

18. Faculty of Nursing, Physiotherapy and Podiatry. Teaching Guides Degree in Nursing (2018). [Internet] University Complutense of Madrid. 2020 [cited 2020 Aug 18]. Available form: https://enfermeria.ucm.es/estudios/grado-enfermeria2020

19. Fisher TD, and Hall RG. A Scale for the comparison of the Sexual Attitudes of Adolescents and their parents. The Journal of Sex Research. 1988; 24: 90-100. Available from: doi: 10.1080/00224498809551400

20. Diéguez Ruibal JL, Castedo AL, Sueiro Domínguez E, and López Sánchez F. Propiedades psicométricas de la escala de actitudes hacia la sexualidad (ATSS) ampliada Cuadernos de medicina psicosomática y psiquiatría de enlace. 2005; 74: 4656. Available form: https://docplayer.es/82922282-Propiedades-psicometricas-de-laescala-de-actitudes-hacia-la-sexualidad-atss-ampliada.html

21. Caron SL, Davis CM, Halteman WA, Stickle M. Predictors of condom-related behaviors among first-year college students. Journal of Sex Research. 1993. 30(3): 252-259. Available from: https://doi.org/10.1080/00224499309551709

22. Sierra JC, Rojas A, Ortega V, Martín Ortiz JD. Evaluación de actitudes sexuales machistas en universitarios: primeros datos psicométricos de las versiones españolas de la Double Standard Scale (DSS) y de la Rape Supportive Attitude Scale (RSAS). International Journal of Psychology and Psychological Therapy. 2007; 7:41-60. Available from: https://dialnet.unirioja.es/servlet/articulo?codigo $=2305078$

23. Díaz Rodríguez I, Gil Llario MD, Morell Mengual V, Salmerón Sánchez P, \& Ruiz Palomino E. Actitudes hacia la sexualidad: ¿difieren las personas con discapacidad intelectual de la población general. International Journal of Developmental and Educational Psychology. 2016; 1(1): 235-242 Available form: https://www.redalyc.org/pdf/3498/349851776027.pdf

24. Diéguez, J, Sueiro, E, López, F. Actitudes y conductas sexuales [Doctoral dissertation] Vigo: Universidad de Vigo; 2007. Available from: https://docplayer.es/23946482-Actitudes-y-conductas-sexuales-dieguez-jl-sueiro-e- 
lopez-f-facultad-de-ciencias-de-la-educacion-universidad-de-vigo-campus-de-

ourense.html

25. Sierra, JC, amd Gutiérrez-Quintanilla, JR. Estudio psicométrico de la versión salvadoreña de la Double Standard Scale. Cuadernos de Medicina Psicosomática y Psiquiatría de Enlace. 2007; 83: 23-30. Available from: http://www.editorialmedica.com/editorialmedica publicacion numero detalle.php?nid= 282\&t=cuadernos\&y=2007\&m=Julio-Agosto-Septiembre\%202007\&num=83

26. Alonso-Martínez, Laura; Heras-Sevilla, Davinia; Fernández-Hawrylak, María. Creencias sobre la Educación Afectivo Sexual en los estudiantes de Ciclos Formativos sanitarios y sociosanitarios. Evidentia. 2021; 18: e12825. Disponible en: http://ciberindex.com/c/ev/e12825

27. Sanz-Martos S, López-Medina IM, Álvarez-García C, Clavijo-Chamorro MZ, Ramos-Morcillo AJ, López-Rodríguez MM, et al. Young Nursing Student's Knowledge and Attitudes about Contraceptive Methods. International Journal of Environmental Research and Public Health [Internet]. 2020;17(16):5869. Available from: http://dx.doi.org/10.3390/ijerph17165869

28. Sharon D, Gonen A, Linetsky I. Factors influencing nursing students' intention to practice sexuality education in their professional work. American Journal of Sexuality Education. 2020: 15(2), 262-278. Available from: https://doi.org/10.1080/15546128.2020.1724223

29. Kaplan Serin E, Alp Dal N, Ikde Öner, Ö. The Communication Skills and Empathic Tendencies of Nursing and Midwifery Students to Reproductive Pattern Section of the Care Plan. Sex Disability. 2020; 38, 161-171. Available from: https://doi.org/10.1007/s11195-019-09587-2

30. Carvalho MCMP, Queiroz, A B A, Ferreira MA, Moura MAV, Pinto C B, Vieira BDG. Ineffectiveness of information and access to health services: vulnerability to human papillomavirus. International nursing review. 2019; 66(2), 280-289. Available from: https://doi.org/10.1111/inr.12500 